\title{
Original
}

Journal of Hard Tissue Biology 23[1] (2014) 101-110

\section{Regenerative Cartilage made by Fusion of Cartilage Elements derived from Chondrocyte Sheets prepared in Temperature-Responsive Culture Dishes}

\author{
Yoshiyuki Mori ${ }^{1}$, Sanshiro Kanazawa ${ }^{2)}$, Yukiyo Asawa ${ }^{2)}$, Tomoaki Sakamoto ${ }^{2)}$, Ryoko Inaki ${ }^{1}$, Kazumi Okubo ${ }^{1)}$, \\ Satoru Nagata ${ }^{3)}$, Makoto Komura ${ }^{4)}$, Tsuyoshi Takato ${ }^{1)}$ and Kazuto Hoshi ${ }^{1,2)}$
}

\begin{abstract}
1) Department of Sensory and Motor System Medicine, Graduate School of Medicine, The University of Tokyo, Tokyo, Japan ${ }^{2)}$ Department of Cartilage \& Bone Regeneration (Fujisoft), Graduate School of Medicine, The University of Tokyo, Tokyo, Japan

3) NAGATA Microtia and Reconstructive Plastic Surgery Clinic, Saitama, Japan

4) Department of Pediatric Surgery, Faculty of Medicine, Saitama Medical University, Saitama, Japan

(Accepted for publication, December 11, 2013)
\end{abstract}

\begin{abstract}
To establish a large regenerative cartilage without any scaffolds, we cultured chondrocytes on NIsopropylacrylamide (NIPPAM)-coated dishes with $3 \times 3 \mathrm{~mm}^{2}$ grids, producing many cell sheets. We then attempted to have the cell sheets form into pellets, which we term cartilage elements, and have the cartilage elements fuse with each other. Finally, we regenerated the large cartilaginous constructs with some stiffness. The NIPPAM coating on culture dishes, which enables to detach cultured chondrocytes only by the decrease in incubation temperature, was useful for preparing many chondrocyte sheets with a homogenous size. The cell sheets could provide effective matrix production and become spherical to form the cartilage elements. For their fusion, those elements were jammed into an agarose mold and were cultured for 3 weeks. The regenerative constructs made by fusing the cartilage elements derived from human or beagle chondrocytes were subcutaneously transplanted into nude mice and the cell-donor beagles, respectively, showing fair cartilage regeneration 2 months after transplantation. They could also prevent severe foreign-body reactions that often occur when using some scaffolds. Thus, by mass production of homogenous cartilage elements using the NIPPAM-coated dishes and their mutual fusion in the agarose mold, mature cartilage was three-dimensionally regenerated without any scaffolds.
\end{abstract}

Key words: Temperature-responsive, Culture dish, Chondrocyte, Tissue engineering, Three dimension

\section{Introduction}

Cartilage exists in ears, nose, trachea, or joints, as a major connective tissue. It plays important roles in maintaining shapes or smooth movement of body. For examples, the cartilage forms a complicated ear shape, arch of ala nasi, and smooth joint surface. However, it lacks a self-repairing capacity. Once damaged, it is unlikely to be naturally regenerated ${ }^{1)}$. As specific shapes in cartilage are strongly involved in biological functions, regenerating three-dimensionally-shaped cartilaginous tissue is important in treating diseases associated with cartilage.

We regenerated cartilage by administering cultured auricular chondrocytes into poly L-lactic acid (PLLA) porous scaffold ${ }^{2}$. The rigidity of the PLLA porous scaffold facilitated the formation of a three-dimensional structure. We established a technique for shaping the PLLA porous scaffold like human nose and making

Correspondence to: Dr. Kazuto Hoshi, Department of Cartilage and Bone Regeneration (Fujisoft), Graduate School of Medicine, The University of Tokyo, Hongo 7-3-1, Bunkyo-ku, Tokyo, 113-8655 Japan; Tel.: +813-3815-9891; Fax: +81-3-5800-9891; E-mail: pochi-tky@umin.net tissue-engineered cartilage with that scaffold, and introduced it into clinical practice to treat nasal deformity of cleft lip and palate ${ }^{1}$. Many other research groups had been also attempted to use biodegradable polymers ${ }^{3-5)}$. However, most biodegradable polymers are biologically absorbed by macrophages, causing foreign-body reactions ${ }^{6,7)}$. Although we chose the PLLA scaffold in the medical introduction as those tissue reactions were relatively low, especially at the early stage of transplantation ${ }^{8)}$, those tissue reactions were somewhat inevitable even in the PLLA scaffold.

Thus, a technique to produce three-dimensional regenerative cartilage without using a scaffold will provide safer and more reliable regenerative medicine for cartilage. However, for this purpose, matrix production by chondrocytes must be strongly enhanced in vitro to increase mechanical strength. Currently, cell aggregation (pellet formation) has been employed to induce cartilage matrix production in vitro ${ }^{9,10)}$. Those reports described that an aggregate of approximately 200,000 cells were cultured in a conical tube to prepare a small mass of $1 \mathrm{~mm}$. As in such a case that is suffering from cleft-lip nose, approximately $1 \mathrm{~mL}$ of 
regenerative cartilage is required ${ }^{1)}$, more than 1,000 tubes should be prepared to regenerate the cartilage, which is practically difficult in manual preparation.

Therefore, we investigated the use of temperature-responsive culture dishes. In those dishes, N-Isopropylacrylamide (NIPPAM) is coated to make a temperature-responsive system ${ }^{11}$. As the dish becomes hydrophillic at low temperature, cultured cells detach automatically. Thus, a cell sheet can be made with cell-to-cell or cell-matrix adhesion maintained ${ }^{12)}$. If NIPPAM-coated regions were patterned into small compartments on the bottom surface of a large culture dish, lowering the temperature facilitates the simultaneous production of many small cell sheets.

In the present study, chondrocytes were cultured on a dish of $6 \mathrm{~cm}$ diameter, with $3 \times 3 \mathrm{~mm}^{2}$ NIPPAM-coat regions patterned, producing many $3 \times 3 \mathrm{~mm}^{2}$ cell sheets. In addition, we attempted cell sheets to be formed into pellets, which we term cartilage elements, through cell-to-cell adhesion, leading to matrix production in chondrocytes. Those cartilage elements were then made to fuse with each other by sharing the matrices the chondrocytes produced, finally regenerating a larger mass of cartilaginous constructs. The purpose of the present study was to establish a method of regenerating a large mass of cartilaginous construct without using any scaffolds, in order to contribute to the development of a novel technique for regenerative medicine.

\section{Materials and Methods}

\section{Chondrocyte culture}

All experimental procedures were approved by the Ethics Committee of the University of Tokyo Hospital (approval number: 622) and Animal Experiment Committee of the University of Tokyo Graduate School of Medicine (approval number: P09-026). Human chondrocytes were isolated from a microtia patient undergoing surgery of the ear. After obtaining informed consent, the perichondrium was detached from about 2-3 $g$ of excised residual auricular cartilage, and the cartilage was cut into $1 \mathrm{~mm}^{3}$ pieces using a scalpel. These pieces were incubated in $0.15 \%$ collagenase solution at $37^{\circ} \mathrm{C}$ for $24 \mathrm{~h}$ in a thermostat with shaking. The lysate was filtered through a cell strainer with a $100 \mu \mathrm{m}$ pore size. After removing the residue, the filtrate was centrifuged at $500 \times \mathrm{g}$ for $5 \mathrm{~min}$, and human auricular chondrocytes were isolated. The isolated chondrocytes were seeded in a $100 \mathrm{~mm}$ collagencoated plastic tissue culture dish (AGC Techno Glass, Chiba, Japan) at a density of 2,500 cells $/ \mathrm{cm}^{2}$, and cultured in chondrocyte growth medium (DMEM/F12 containing 5\% human serum, 100 $\mathrm{ng} / \mathrm{ml} \mathrm{FGF-2,} \mathrm{and} 5 \mu \mathrm{g} / \mathrm{ml}$ insulin) ${ }^{13,14)}$ in a $37{ }^{\circ} \mathrm{C} / 5 \% \mathrm{CO}_{2}$ incubator. The medium was changed twice a week. Before reaching confluence, the cells were treated with trypsin-EDTA solution and passaged. The Cells were used in experiments after 2 passages.

Canine chondrocytes were isolated from the auricle of a beagle ( 8 months old, body weight: approximately $10 \mathrm{~kg}$ ). The anesthesia was intravenously induced by $1 \mathrm{mg} / \mathrm{kg}$ propofol and maintained with $2 \%$ halothane in inhalation. The unilateral auricular cartilage (approximately 2-3 g) of the beagles was harvested by an aseptic surgical treatment. The perichondrium was exfoliated from the native cartilage using a scalpel, and then the cartilage was minced to a size of $1 \mathrm{~mm}^{3}$. The fragments of cartilage were incubated for collagenase digestion in a $0.15 \%$ collagenase solution at $37{ }^{\circ} \mathrm{C}$ in $24 \mathrm{~h}$. The collected cells counted after dyeing with trypan blue were confirmed for cell viability. The isolated $2.0 \times 10^{5}$ chondrocytes were seeded in the $100 \mathrm{~mm}$ collagen-coated dish and cultured in DMEM/F12 containing $5 \%$ autologous serum, $100 \mathrm{ng} / \mathrm{ml} \mathrm{FGF}-2$, and $5 \mu \mathrm{g} / \mathrm{ml}$ insulin in a $37{ }^{\circ} \mathrm{C} / 5 \% \mathrm{CO}_{2}$ incubator ${ }^{13,14)}$. In the prepapration of autologous serum, whole blood was harvested from the cervical vein of each beagle, and was allowed to clot over $5 \mathrm{~h}$ at room temperature ${ }^{13)}$. The serum was separated by centrifugation of the clotted blood at $500 \times \mathrm{g}$ for 30 minutes. The medium was changed two times per week. Passages were performed by treatment with a trypsin-EDTA solution when the cells were approaching confluence. The cells were used in experiments after 2 passages.

The chondrocytes isolated from both species were seeded in NIPPAM-coated dishes with $3 \times 3 \mathrm{~mm}^{2}$ grids $(\varphi 6 \mathrm{~cm}$, RepCell, CellSeed, Tokyo, Japan) at $1 \times 10^{5}$ cells per dish, and cultured in the above growth culture medium in a $37{ }^{\circ} \mathrm{C} / 5 \% \mathrm{CO}_{2}$ incubator. The medium was changed twice a week. The dishes were then cooled to $20^{\circ} \mathrm{C}$, and cells were detached with mild vibration. In the control experiment, cells were cultured in normal culture dishes ( $\varphi 6 \mathrm{~cm}$, with or without the grids: Corning, Tewksbury, MA, USA). The cells were detached from the dishes by treatment with trypsin-EDTA solution. Dishes were coated with gelatin as needed. The culture dishes were coated with gelatin. Gelatin (Type A, Sigma-Aldrich, St Louis, MO, USA) was diluted to $0.1 \%$ with distilled water. One $\mathrm{mL}$ of the gelatin solution was poured to the $6 \mathrm{~cm}$ culture dishes. The dishes were left at $37^{\circ} \mathrm{C}$ for $30 \mathrm{~min}$, and then, the solution was aspirated.

\section{Agarose mold}

The cells were detached from the culture dish by lowtemperature or trypsin-EDTA treatment, and carefully transferred to a $15 \mathrm{ml}$ conical tube using a micropipette. The cells were centrifuged at $460 \times \mathrm{g}$ for $5 \mathrm{~min}$, resuspended with $10 \mathrm{~mL}$ of medium containing $5 \%$ serum, insulin, and FGF-2, and transferred to a low-attachment culture dish $(\varphi 10 \mathrm{~cm}$, Corning) to stabilize cell aggregates. After one-day culture in the low-attachment culture dish, the cells were collected in a $50 \mathrm{~mL}$ conical tube, centrifuged at $460 \times \mathrm{g}$ for 5 minutes, and carefully transferred to an agarose mold.

Agarose molds were prepared according to the previous report ${ }^{15)}$. Agarose (Takara Bio, Otsu, Japan) was mixed with MEM at $2 \%$ and dissolved at $121^{\circ} \mathrm{C}$. As an agarose mold for human 

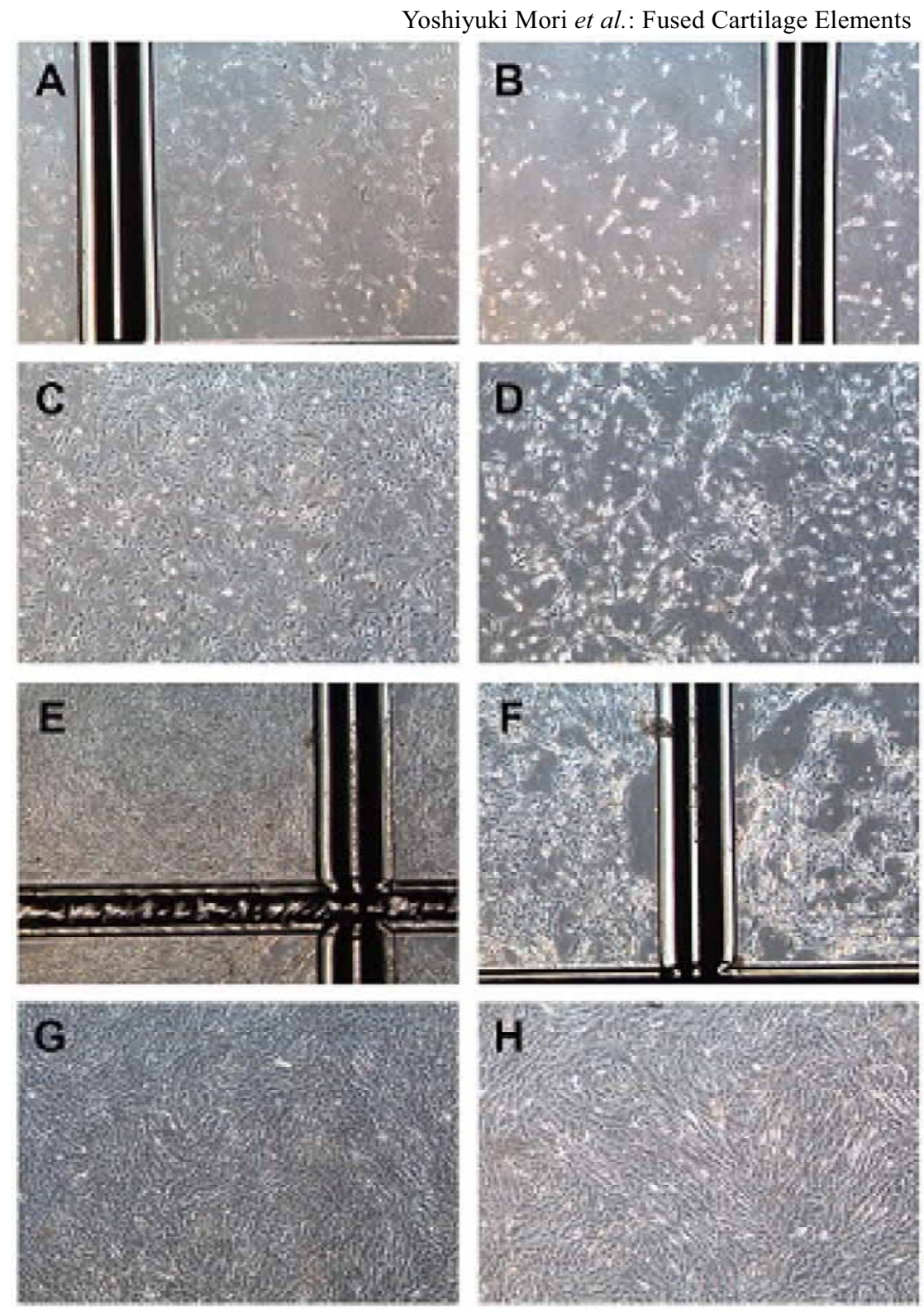

Figure 1. Conditions for chondrocyte culture using the NIPPAM-coated dishes. Gelatin coating promoted chondrocyte adhesion on cell seeding and prevented from unpredictable detachement during cell culture. Macroscopic findings: A and B; Binocular appearance: $\mathrm{C}$ and $\mathrm{D}$; After fusion: $\mathrm{E}, \mathrm{F}, \mathrm{G}$ and $\mathrm{H}$ : NIPPAM coating $(+)$ : A, C, E and G, (-), B, D, F and H. cells, $8 \mathrm{ml}$ of $2 \%$ agarose solution sterilized using an autoclave was added to a well of 6-well culture plate (Agc Techno), and a $15 \times 5 \times 5 \mathrm{~mm}$ concavity was made. Regarding the agarose mold for beagle cells, $80 \mathrm{~mL}$ of $2 \%$ agarose solution sterilized using an autoclave was added to a $\varphi 10 \mathrm{~cm}$ glass vessel, and a $50 \times 5 \times 5$ mm concavity was made.

\section{Transplantation to nude mice}

Human chondrocytes of five culture dishes $(\varphi 6 \mathrm{~cm})$ were used to prepare a transplant for nude mice. The human chondrocytes from the five culture dishes were transferred to the agarose mold and cultured for 3 weeks in $8 \mathrm{ml}$ of differentiation medium containing BMP-2, insulin, and $\mathrm{T} 3{ }^{10}$. The medium was changed twice a week. The region around the concavity in the agarose mold was then cut out and was used to transplant the cells to subcutaneous space in a Bulb-c nude mouse (6 w, male). Transplantation was performed under nembutal anesthesia. Two $\mathrm{cm}$ incision was made in the dorsal skin, and the skin was detached. The transplant was put into the subcutaneous space by placing the agarose mold in the subcutaneous pocket, and excised after 2 months. When it was excised, the animal was euthanized with nembutal anesthesia. The sample was excised and photographed, and then subjected to histological analysis. This experiment was performed 3 times using 3 lots of human chondrocytes and 3 nude mice.

\section{Transplantation to beagles}

Canine chondrocytes of 20 culture dishes $(\varphi 6 \mathrm{~cm})$ were used to prepare a transplant for beagles. The cells of the 20 culture dishes were transferred to the agarose mold for beagles and cultured for 3 weeks in $80 \mathrm{~mL}$ of mediumcontaining $5 \%$ autologous canine serum, insulin, and FGF- $2^{14)}$. The medium was changed twice a week. The region around the concavity in the agarose mold was then cut out and transplanted to the subcutaneous 

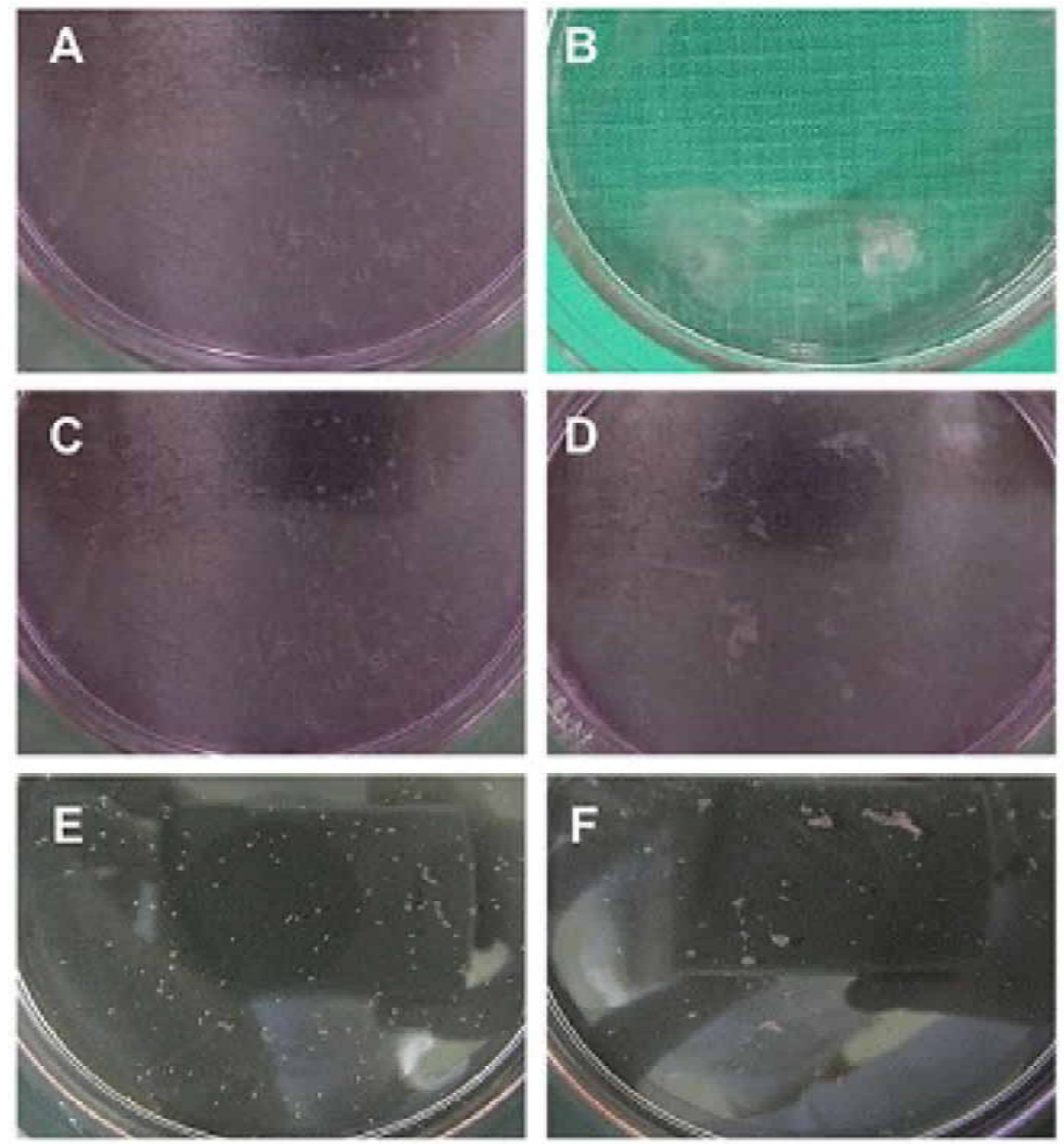

Figure 2. Many chondrocyte sheets were prepared to form homogenous cartilage elements. The chondrocytes cultured under the monolayer condition were made to be detached from culture dishes with lowtemperature treatment ( $\mathrm{A}, \mathrm{C}$ and $\mathrm{E})$ or enzyme treatment (B, D and F). After detachment of cells, the cell sheets were transferred into the low-attachment dishes. Immediately after detachment: $\mathrm{A}$ and $\mathrm{B}$; Day 0 of non-adhesive culture: $\mathrm{C}$ and $\mathrm{D}$; Day 1: E and F.

space in the cell-donor beagle. For the transplantation of the tissueengineered cartilage, the anesthesia was inducted in a similar manner. The transplant was placed into subcutaneous pocket, and was operatively harvested after transplantation for 2 months. The sample was excised, photographed, and subjected to histological analysis. This experiment was performed twice using 2 beagles.

\section{Histological evaluation}

The samples were fixed in 4\% paraformaldehyde solution $/ 0.1$ M phosphate buffer ( $\mathrm{pH} \mathrm{7.4)}$ at $4{ }^{\circ} \mathrm{C}$ for $2 \mathrm{~h}$, and immersed in 10 $\%$ sucrose/PBS and $20 \%$ sucrose/PBS, sequentially. They were put in 1:2 mixtures of OCT compound and $20 \%$ sucrose/PBS at 4 ${ }^{\circ} \mathrm{C}$ overnight. The samples were then rapidly frozen in liquid nitrogen. Frozen sections (10 $\mu \mathrm{m}$ thickness) were prepared using a microtome (CM1850-Kryostat, LEICA), stained with toluidine blue-O and hematoxylin-eosin, and observed under a light microscope (Olympus DP 70, Tokyo, Japan).

\section{Results \\ Preparation of cartilage elements}

In order to prepare many cartilage elements, we attempted to use the NIPPAM-coated dishes. First of all, we examined necessity of additional gelatin coating that enhanced cell adhesion in cell seeding. In the NIPPAM-coated dishes, cell adhesion seemed less than in the conventional dishes 2 days after seeding, regardless of the presence or absence of a gelatin coat (Fig. 1). However, favorable cell adhesion and growth were noted at 6 days of culture using the NIPPAM coating with the additional gelatin coating, although in the absence of gelatin coat, some cells were detached from the NIPPAM-coated dishes (Fig. 1, F). Thereafter, we added gelatin coating when we used NIPPAM-coated dishes for chondrocytes.

Next, we investigated the usefulness of the NIPPAM coating on preparing homogenous cartilage elements (Fig. 2). In the NIPPAM-coated dishes with $3 \times 3 \mathrm{~mm}^{2}$ grids, homogenous chondrocyte sheets fitting to the grid size ( $3 \mathrm{~mm}$ square) were produced immediately after detachment by low-temperature treatment (Fig. 2, A), although in the culture dishes with the grids but without NIPPAM coating, as adhering cells were detached by trypsin-EDTA treatment, the size of detached sheets were heterogeneous (Fig. 2, B). After transfer to low-attachment culture dish, the sheets immediately shrank and became cell aggregates with the same size of approximately $1 \mathrm{~mm}$ at 1 days of culture, forming homogenous cartilage elements (Fig. 2, E). In contrast, in the absence of the NIPPAM coating, the size of detached sheets or cartilage elements was not constant (Fig. 2, F). 
Yoshiyuki Mori et al.: Fused Cartilage Elements
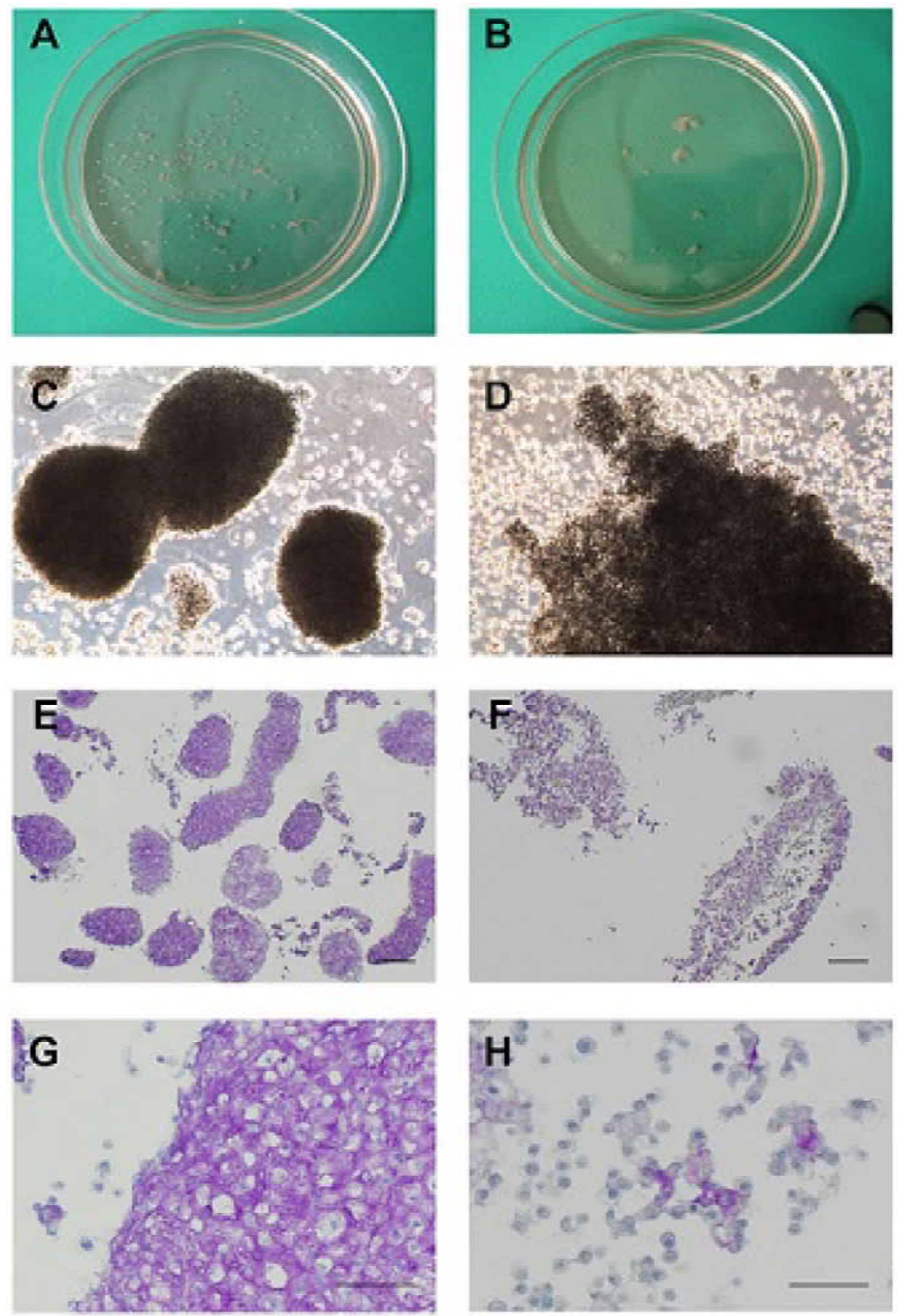

Figure 3. The cartilage elements made of human auricular chondrocytes. The chondrocyte sheets prepared with (A, C, E and G) or without (B, D, F and $\mathrm{H})$ the NIPPAM-coated dishes were transferred into the low-attachment culture dishes, and were incubated for three weeks. After the incubation, the cartilage elements prepared with the NIPPAM-coated dishes showed metachromagia in toluidinen blue staining, suggesting accumulation of cartilage matrices. Macroscopic findings: A and B; Binocular appearance: $\mathrm{C}$ and $\mathrm{D}$; Histology: E, F, G and H. Bar=1 mm (E and $\mathrm{F})$ and $100 \mu \mathrm{m}(\mathrm{G}$ and $\mathrm{H})$.

In order to confirm the matrix production of cartilage elements, we compared the efficacy on matrix production between homogenous cartilage elements made in the NIPPAM-coated dishes and heterogeneous ones by enzyme treatment (Fig. 3). Three weeks' incubation induced abundant matrix production of cartilage in homogenous cartilage elements made in the NIPPAM-coated dishes (Fig. 3, A, C, E, G). In contrast, when the elements were prepared by enzyme treatment, relatively large aggregates were scattered, while the chondrocytes were dispersed inside the large aggregates and matrix production was hardly observed (Fig. 3, B, D, F, H). Therefore, the NIPPAM coating was useful to prepare many cartilage elements with a homogenous size and effective matrix production.

\section{Fusion of cartilage elements}

The production of large-sized regenerative cartilage was attempted, by fusing cartilage elements (Fig. 4). Those elements were jammed into the agarose mold, and were incubated for matrix production and subsequent adhesion during 3 weeks. The cartilage elements of human chodrocytes prepared in both of the NIPPAMcoated dishes and the conventional dishes were put in the agarose molds to regenerate about $1.5 \mathrm{~cm}$ rod-shaped cartilage. After 3 weeks of incubation, the shapes of the regenerative constructs from the cartilage elements prepared in the NIPPAM-coated dishes were favorable, and the rod shape was retained even when the constructs were removed from the agarose mold (Fig. 4, A, C, E, $\mathrm{G})$. In contrast, the aggregates of the cartilage elements prepared 

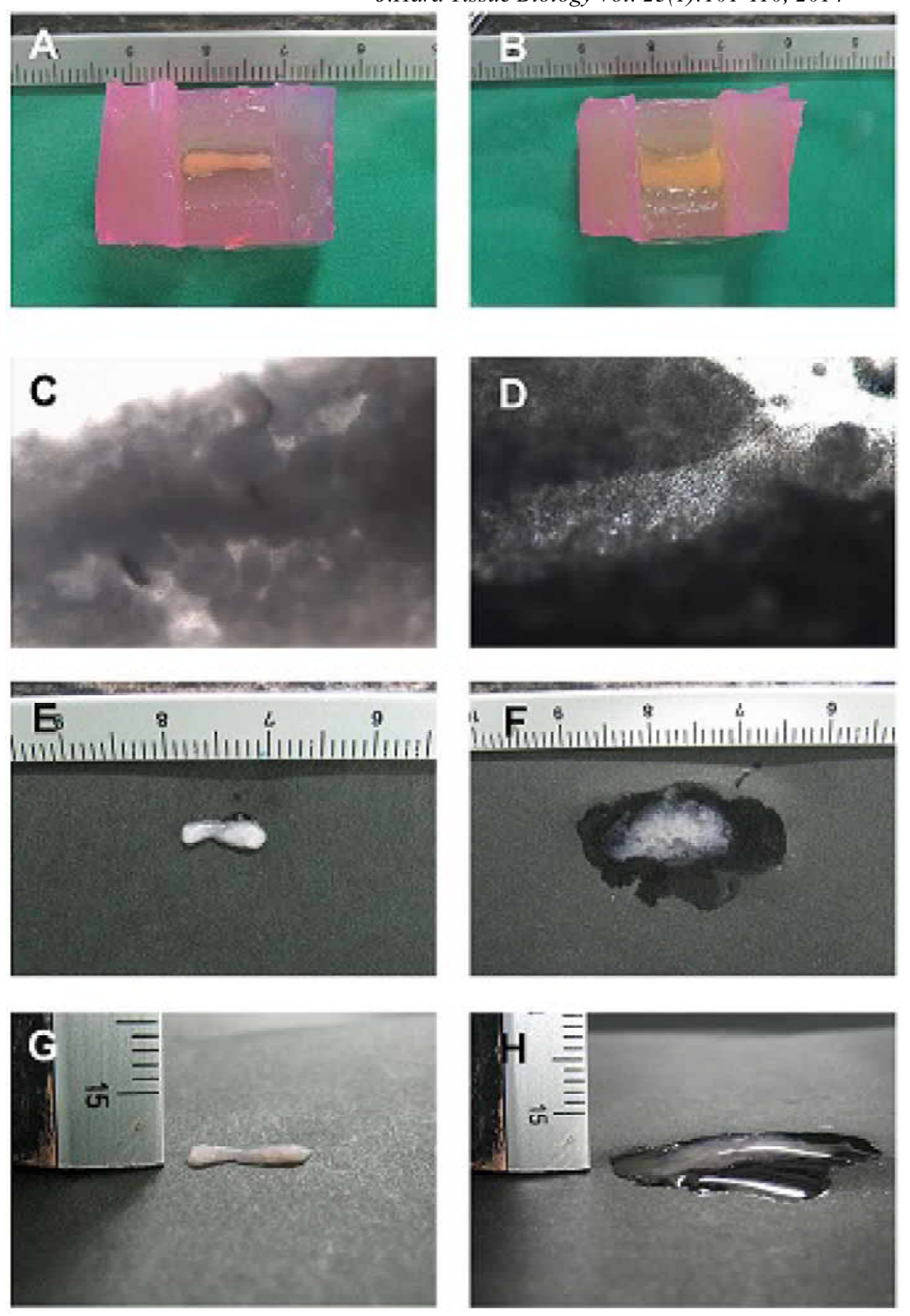

Figure 4. The regenerative constructs by fusing the cartilage elements made of human auricular chondrocytes. The cartilage elements derived from the chondrocytes prepared in the NIPPAM-coated dishes (A, C, E and G) was compared on with those prepared in the conventional dishes (B, C, F and H). In the binocular appearance $(\mathrm{C}$ and $\mathrm{D})$, each cartilage element, like a pellet, was visible in NIPPAM coat (C). After fusion (E, $\mathrm{F}, \mathrm{G}$ and $\mathrm{H}$ ), which was done by the incubation in the agarose mold for three weeks, a rod shape was kept in the regenerative constructs using the NIPPAM-coated dishes (E and G). Macroscopic findings: A and B.

in conventional dishes dispersed immediately after removal from the agarose mold, and the rod-shape could not be maintained (Fig. 4, B, D, F, H).

\section{Transplantation of regenerative constructs made by fusion of cartilage elements}

When the regenerative constructs $(1.5 \mathrm{~cm}$ long $)$ made by fusion of the cartilage elements using human chondrocytes cultured in the NIPPAM-coated dishes were subcutaneously transplanted into nude mice (Fig. 5, A), favorably regenerative cartilage was noted 2 months after transplantation (Fig. 5, B-F). On histological evaluation of the regenerative constructs, abundant metachromatic matrix was present around chondrocytes, showing favorable cartilage regeneration (Fig. 5, E, F).

The similar experiment was performed in beagles. Beaglederived auricular chondrocytes were cultured in the NIPPAMcoated dishes, formed the cartilage elements, and were made to be the regenerative constructs in the agarose mold (Fig. 6, A). The regenerative constructs with the rod shape of $5 \mathrm{~cm}$ were autologously transplanted into the subcutaneous space of beagle back. When the regenerative constructs were excised 2 months after transplantation, the length of constructs became shorter (Fig. 6, B-D). However, the constructs were sufficiently stiff and the shape was rather retained (Fig. 6, C, D). Histologically, abundant extracellular matrix was found around the round cells (Fig. 6. E). Those matrices were stained in metachromagia with toluidine blue 

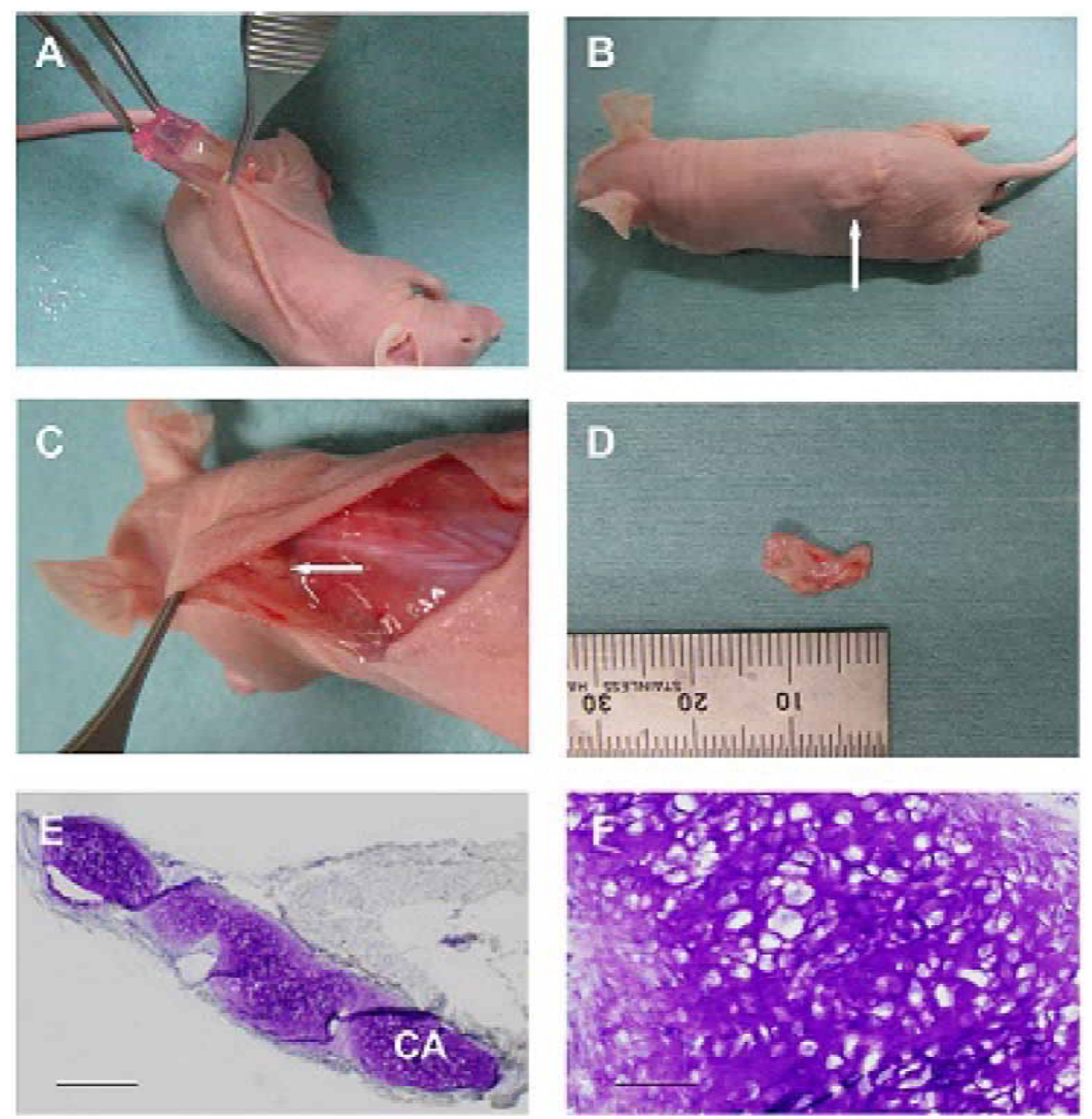

Figure 5. The regenerative constructs by fusing cartilage elements were made of human auricular chondrocytes and were transplanted into nude mice.

A. The regenerative constructs were transplated into the subcutenous space of nude mice, using agarose mold.

B. The transplants could be detected though the skin of nude mice (arrow), 2 months after transplantation.

C. The regenerative construct by fusing cartilage elements (arrow), transplanted into nude mice was removed from subcutaneous space 2 months after transplantation.

D. The size of regenerative construct was almost $1.5 \mathrm{~cm}$ long after transplantation, which was similar than what had been before transplantation.

E. The histological findings of the regenerative constructs after transplantation in toluidine blue staining showed that metachromatic areas, suggesting cartilaginous tissues, were accumulated (CA). Bar $=1 \mathrm{~mm}$.

F. High magnification of the histological findings in toluidine blue staining. Metachromatic areas were observed in extracellular matices, showing the accumulation of proteoglycans. Bar $=100 \mu \mathrm{m}$.

Those experiments were triplicated, showing the similar results.

staining, suggesting favorable cartilage regeneration (Fig. $6 \mathrm{~F}$ ).

\section{Discussion}

The NIPPAM-coated dishes can detach adhesive cells not by enzymatic treatment, but just by low-temperature treatment. In the preparation using the NIPPAM-coated dishes, both cell-tocell adhesion molecule and extracellular matrix, all of which are pivotal for maintaining cell functions, are well preserved ${ }^{11,12}$. The chondrocytes that were used in the present study are typical adhesive cells. When the chondrocytes are suspended as cell sheets, they are three-dimensionally adhered to themselves directly or via extracellular matrices including collagen, fibronectin, or proteoglycans, thereby forming pellets.

Cultured chondrocytes hardly produce cartilage matrices under the condition of monolayer culture, but begin to effectively produce the cartilage matrix when they form pellets ${ }^{10}$. During cartilage development, mesenchymal stem cells aggregate and differentiate into chondrocytes ${ }^{16)}$. As the cultured chondrocytes are known to resemble mesenchymal stem cells in the aspects of gene expression pattern or differentiation ability ${ }^{17)}$, the pellet culture of mesenchymal stem cells or cultured chondrocytes may reproduce physiological chondrocyte differentiation ${ }^{9,10)}$. Therefore, the 

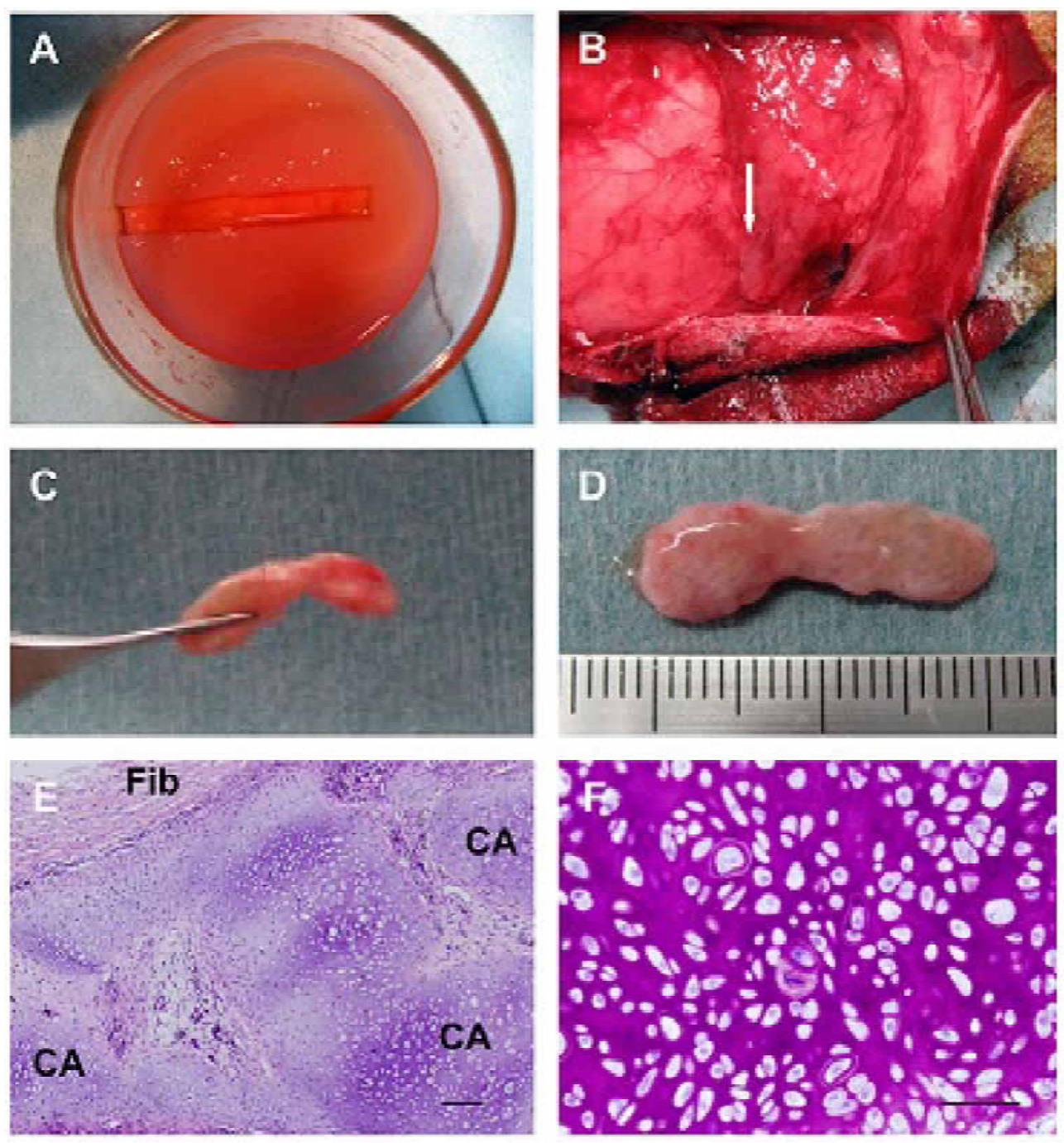

Figure 6. Autologous transplantation of the regenerative constructs by fusing cartilage elements into beagles

A. The cartilage elements of beagle auricular chondrocytes derived from total twenty NIPPAM-coated dishes were placed into agarose mold and were incubated for three weeks.

B. The regenerative construct (arrow), autologously transplanted into beagles, was removed from subcutaneous space 2 months after transplantation.

C. The regenerative construct after transplantation was sufficiently stiff.

D. The size of regenerative construct was almost $2 \mathrm{~cm}$ long after transplantation, which was rather shorter than what had been before transplantation $(5 \mathrm{~cm})$.

E. The histological findings of the regenerative constructs after transplantation in hematoxylene and eosin staining showed that cartilaginous areas were accumulated (CA), surrounded by fibours tissues (Fib). Bar $=100 \mu \mathrm{m}$.

F. High magnification of the histological findings in toluidine blue staining. Metachromatic marices surrounded chondrocytes, suggesting the accumulation of proteoglycans. $B a r=100 \mu \mathrm{m}$.

Those experiments were duplicated, showing the similar results.

application of pellet culture, which was termed cartilage elements in this study, for the promotion of cartilage maturation was regarded to be reasonable.

The size of cartilage elements was set to approximately $1 \mathrm{~mm}$ in the present study. Undifferentiated mesenchymal stem cells and prechondrocytes seem more sensitive to hypoxia or malnutrition than mature chodrocytes ${ }^{10}$. In the previous reports, the sizes in pellets of mesenchymal stem cells and their analog, cultured chodrocytes, were usually confined within $1 \mathrm{~mm}$, because larger pellets caused necrosis. The chondrocyte sheets of $3 \mathrm{~mm}$ square
(10-100 $\mu \mathrm{m}$ of thickness) became a pellet of approximately $1 \mathrm{~mm}^{3}$ (i.e., a sphere of $1 \mathrm{~mm}$ ) in volume. Therefore, we applied the NIPPAM-coated temperature-responsive culture dishes patterned with $3 \mathrm{~mm}$ grids to produce a large amount of homogenous cell sheets, which was advantageous in mass production of cartilage elements to facilitate in vitro matrix production.

In order to produce a larger three-dimensional regenerative cartilage, these cartilage elements should be fused with each other. Because concentrating a large amount of cells into one place at a high density requires improvements in substance exchange, the 
Yoshiyuki Mori et al.: Fused Cartilage Elements

agarose mold was employed ${ }^{15)}$. Agarose excels at substance permeability and the diffusion of water, nutrients, growth factors, and wastes. Shaping a template to prepare an agarose mold also allows the formation of any three-dimensional regenerative cartilage. In addition, local stress to the regenerative constructs that might be loaded when they are just immersed in culture medium can be prevented, because the agarose mold can support the weight of constructs on curved surface. Thus, the agarose mold contributed to fusing the cartilage elements and to forming the large-sized regenerative constructs effectively.

In order to facilitate to fuse the cartilage elements, we used the cocktail medium of BMP-2, insulin, and T3 that strongly induces matrix production in cultured human chondrocytes ${ }^{10}$. Cartilage matrices mainly consist in type II collagen and proteoglycan. These molecules are associated with each other through complicated and irregular intersections ${ }^{18)}$, and are chemically bound to other matrix proteins (e.g., tenascin and fibronectin), to be polymerized ${ }^{19}$. Finally, these molecules can be also anchored to adjacent chondrocytes through adhesion molecules, such as CD44 and integrin ${ }^{20)}$. Thus, we speculated that the cartilage elements were fused by promoting matrix production in the chondrocytes.

However, the effects of this culture medium on matrix production in canine chondrocytes seemed rather smaller, compared with those in human chondrocytes that had been confirmed by the previous study ${ }^{10}$. In the present study, the beagle chondrocytes were cultured in a medium containing autologous serum. The reason why the regenerative constructs of $5 \mathrm{~cm}$ became reduced in size after autologous transplantation into beagles may be explained by the low level of matrix production before transplantation due to no use of the cocktail medium.

Such regenerative constructs by fusing cartilage elements were transplanted in vivo to obtain mature cartilaginous tissues. In the regenerative constructs transplanted into nude mice or immunologically normal beagles, neither the excessive infiltration of macrophages nor severe foreign-body reaction was observed. Up to date, some scaffolds made of biodegradable polymers have been needed to fabricate three-dimensional regenerative tissues, but severe tissue foreign body reactions were evoked after transplantation ${ }^{21}$. Although the use of biodegradable polymers that show low speed of biodegradation could reduce the foreignbody reactions under the in vivo conditions ${ }^{8)}$, it is hard to eliminate this adverse reaction, as far as we use any biodegradable polymers as scaffolds. Thus, an effective method to regenerate threedimensional tissues and also to minimize foreign-body reactions after transplantation should be established. In the present study, by mass production of homogenous cartilage elements and their mutual fusion, mature cartilage was three-dimensionally regenerated without any scaffolds. However, the mechanical strength of regenerative constructs by fusing cartilage elements without using any scaffolds may not be sufficient to maintain appropriate three-dimensional shapes after transplantation. The reinforcement of mechanical strength in regenerative cartilage before transplantation is an important issue to improve the properties of tissue-engineered cartilage and to widen its clinical indication. Further studies are needed to promote matrix production of cultured chondrocytes, which can greatly improve this challenging method to make many cartilage elements fuse into a large-sized regenerative cartilage without using any scaffolds.

\section{Acknowledgements}

We thank Ms. Miki Akizawa, Mr. Makoto Watanabe and Ms. Yuko Motoki for technical support. This work was supported by Grants-in-Aid for Scientific Research from the Ministry of Education, Culture, Sports, Science and Technology of Japan (MEXT, No. 24390451, 24593050 and 25670847), and Research and Development Programs for Self-maturating Device from the New Energy and Industrial Technology Development Organization.

\section{References}

1. Hoshi K, Fujihara Y, Asawa Y, Nishizawa S, Kanazawa S, Sakamoto T, Watanabe M, Ogasawara T, H S, Mori Y and Takato T. Recent trends of cartilage regenerative medicine and its application to the oral and maxillofacial surgery. Oral Sci Int 10: 15-19, 2013

2. Tanaka Y, Yamaoka H, Nishizawa S, Nagata S, Ogasawara T, Asawa Y, Fujihara Y, Takato T and Hoshi K. The optimization of porous polymeric scaffolds for chondrocyte/atelocollagen based tissue-engineered cartilage. Biomaterials 31: 45064516, 2010

3. Ushida T, Furukawa K, Toita K and Tateishi T. Threedimensional seeding of chondrocytes encapsulated in collagen gel into PLLA scaffolds. Cell Transplant 11: 489494, 2002

4. Hutmacher DW, Ng KW, Kaps C, Sittinger M and Klaring S. Elastic cartilage engineering using novel scaffold architectures in combination with a biomimetic cell carrier. Biomaterials 24: 4445-4458, 2003

5. Hannouche D, Terai H, Fuchs JR, Terada S, Zand S, Nasseri BA, Petite H, Sedel L and Vacanti JP. Engineering of implantable cartilaginous structures from bone marrowderived mesenchymal stem cells. Tissue Eng 13: 87-99, 2007

6. Fujihara Y, Asawa Y, Takato T and Hoshi K. Tissue reactions to engineered cartilage based on poly-L-lactic acid scaffolds. Tissue Eng Part A 15: 1565-1577, 2009

7. Fujihara Y, Takato T and Hoshi K. Immunological response to tissue-engineered cartilage derived from auricular chondrocytes and a PLLA scaffold in transgenic mice. Biomaterials 31: 1227-1234, 2010 
8. Asawa Y, Sakamoto T, Komura M, Watanabe M, Nishizawa S, Takazawa Y, Takato T and Hoshi K. Early stage foreign body reaction against biodegradable polymer scaffolds affects tissue regeneration during the autologous transplantation of tissue-engineered cartilage in the canine model. Cell Transplant 21: 1431-1442, 2012

9. Sekiya I, Colter DC and Prockop DJ. BMP-6 enhances chondrogenesis in a subpopulation of human marrow stromal cells. Biochem Biophys Res Commun 284: 411-418, 2001

10. Liu G, Kawaguchi H, Ogasawara T, Asawa Y, Kishimoto J, Takahashi T, Chung UI, Yamaoka H, Asato H, Nakamura K, Takato T and Hoshi K. Optimal combination of soluble factors for tissue engineering of permanent cartilage from cultured human chondrocytes. J Biol Chem 282: $20407-$ 20415, 2007

11. Shimizu T, Yamato M, Kikuchi A and Okano T. Twodimensional manipulation of cardiac myocyte sheets utilizing temperature-responsive culture dishes augments the pulsatile amplitude. Tissue Eng 7: 141-151, 2001

12. Kushida A, Yamato M, Konno C, Kikuchi A, Sakurai Y and Okano T. Decrease in culture temperature releases monolayer endothelial cell sheets together with deposited fibronectin matrix from temperature-responsive culture surfaces. J Biomed Mater Res 45: 355-362, 1999

13. Tanaka Y, Ogasawara T, Asawa Y, Yamaoka H, Nishizawa S, Mori Y, Takato T and Hoshi K. Growth factor contents of autologous human sera prepared by different production methods and their biological effects on chondrocytes. Cell Biol Int 32: 505-514, 2008

14. Takahashi T, Ogasawara T, Kishimoto J, Liu G, Asato H, Nakatsuka T, Uchinuma E, Nakamura K, Kawaguchi H, Takato T and Hoshi K. Synergistic effects of FGF-2 with insulin or IGF-I on the proliferation of human auricular chondrocytes. Cell Transplant 14: 683-693, 2005

15. Mori Y, Kanazawa S, Watanabe M, Suenaga H, Okubo K, Nagata S, Fujihara Y, Takato T and Hoshi K. Usefulness of agarose mold as a storage container for three-dimensional tissue-engineered cartilage. Mater Sci Appl 4: 73-78, 2013

16. Tuli R, Tuli S, Nandi S, Huang X, Manner PA, Hozack WJ, Danielson KG, Hall DJ and Tuan RS. Transforming growth factor-beta-mediated chondrogenesis of human mesenchymal progenitor cells involves $\mathrm{N}$-cadherin and mitogen-activated protein kinase and Wnt signaling cross-talk. J Biol Chem 278: 41227-41236, 2003

17. de la Fuente R, Abad JL, Garcia-Castro J, Fernandez-Miguel G, Petriz J, Rubio D, Vicario-Abejon C, Guillen P, Gonzalez MA and Bernad A. Dedifferentiated adult articular chondrocytes: a population of human multipotent primitive cells. Exp Cell Res 297: 313-328, 2004

18. Poole AR, Matsui Y, Hinek A and Lee ER. Cartilage macromolecules and the calcification of cartilage matrix. Anat Rec 224: 167-179,1989

19. Kii I, Nishiyama T, Li M, Matsumoto K, Saito M, Amizuka $\mathrm{N}$ and Kudo A. Incorporation of tenascin-C into the extracellular matrix by periostin underlies an extracellular meshwork architecture. J Biol Chem 285: 2028-2039, 2010

20. Knudson $\mathrm{W}$ and Loeser RF. CD44 and integrin matrix receptors participate in cartilage homeostasis. Cell Mol Life Sci 59: 36-44, 2002

21. Eppley BL, Morales L, Wood R, Pensler J, Goldstein J, Havlik RJ, Habal M, Losken A, Williams JK, Burstein F, Rozzelle AA and Sadove AM. Resorbable PLLA-PGA plate and screw fixation in pediatric craniofacial surgery: Clinical experience in 1883 patients. Plast Reconstr Surg 114: 850856, discussion 857, 2004 